\title{
Developmental Trajectories of Adolescent Overweight/Obesity in China: SES Correlates and Health Consequences
}

Ying Liang ( $\sim$ liang_ying@whu.edu.cn )

Wuhan University https://orcid.org/0000-0002-5426-4453

Yaqiang Qi

Renmin University of China

\section{Research article}

Keywords: China, adolescent overweight/obesity trajectories, SES, self-rated health, CFPS

Posted Date: October 16th, 2019

DOI: https://doi.org/10.21203/rs.2.16132/v1

License: @ (i) This work is licensed under a Creative Commons Attribution 4.0 International License.

Read Full License 


\section{Abstract}

Background: During recent decades, China has experienced rapid growth in economy and also in prevalence of childhood obesity. Given the great importance of adolescence overweight/obesity for future health, and given the relative lack of longitudinal studies on adolescent obesity in developing countries, particularly in China, in current study, we aimed to explore the potential growth trajectories of overweight/obesity among Chinese adolescents and to further examine socioeconomic status predictors and health consequences of these growth trajectories.

Methods: The data were from four waves of panel data from the China Family Panel Studies (CFPS 2010, 2012, 2014, and 2016). For the current study, children aged 10 to 12 from the baseline 2010 sample were selected $(N=1685)$, among whom 1388 were re-interviewed in 2012, 1172 in 2014, and 941 in 2016. We retained a final sample of 800 who had at least three waves of BMI data (i.e. final $N=800$ ). Generalized growth mixture modeling (GGMM) was used as the major analytical strategy.

Results: We found that three types of overweight/obesity developmental trajectories may exist for those Chinese adolescents including a stably normal class, a decreased risk class, and a chronically overweight/obese class. Additionally, we found that higher family income was associated with a lower probability of getting into the chronically overweight/obese class for urban adolescents, but with a higher probability of getting into the same class for rural adolescents. Finally, we found that the adolescents in the chronically overweight/obese group reported significantly lower levels of self-rated health.

Conclusions: There were heterogeneous growth trajectories of Chinese adolescent overweight/obesity. Sustained overweight/obesity during adolescence was predicted by lower family income in urban China but by higher family income in rural China. More targeted and regionalized interventions for children's overweight/obesity in China should be considered. Keywords: China; adolescent overweight/obesity trajectories; SES; self-rated health; CFPS

\section{Background}

With the process of worldwide nutrition transition, overweight and obesity is rapidly spreading among developing countries [1-2]. As the largest developing nation, China has seen a huge surge in overweight and obese population in the past several decades, due to its rapid economic growth and the resultant rising living standard and changing lifestyles [3-4]. In the past few decades, overweight and obesity among Chinese children have increased at an increasingly higher speed [5]. For instance, It has been found that the prevalence rate of overweight/obesity for children aged 6-17 has more than doubled from the years 1991 to 2011 , from $11.7 \%$ to $25.2 \%$ [6]. Given the widely acknowledged long-term 
adverse impact of childhood obesity on adult health, including continuing adulthood obesity, elevated risks of cardiovascular and metabolic diseases and all-cause mortality [7$9]$, it is of great necessity to scrutinize the developmental characteristics and unequal patterns of overweight/obesity among Chinese children and adolescents to find ways to slow down its spreading speed and lessen future disease burdens.

Regarding SES correlates of childhood overweigh/obesity, research using single or repeated cross-sectional data has found that the correlation between socioeconomic status and overweight/obesity varies between developed and developing countries [10]. For children in developed countries, studies have found a predominantly inverse relationship between SES and obesity [11-12]. However, based on relatively limited evidence from developing countries, SES was found to be positively correlated with rates of obesity among both adults and children [13-14]. For Chinese children, researchers have similarly found that the impact of family SES on childhood overweight/obesity is positive and has widened over time [15-16].

More recently in research using panel data and person-centered approach, it has been argued that there are various developmental trajectories of childhood overweight/obesity [17-18]. The majority of these studies reported that the developmental trajectories of childhood obesity were indeed heterogeneous, and there were usually three to four distinct "latent" classes necessary for depicting different growth patterns. This research has found that higher SES background decreases the likelihood of persistent or elevated risk of overweight and obesity over time [19-21]. Meanwhile, this body of longitudinal research has also found that the risky developmental trajectories of childhood obesity is associated with serious synchronous morbidity, such as asthma and lung functioning [22], adolescent metabolic health outcomes (e.g., waist circumference, insulin resistance, and total cholesterol) [23], non-alcohol fatty liver disease [24], and dental health [25].

However, almost all these studies on SES correlates and health consequences of childhood obesity trajectories have focused on developed countries. In light of the persisting rural-urban disparities and rising socioeconomic inequalities [26], it remains poorly 
understood how the developmental characteristics of overweight/obesity among Chinese children vary across different socioeconomic strata and between urban and rural areas. Therefore, we draw on longitudinal data from four waves of the China Family Panel Study (CFPS), a large-scale ongoing nationally representative survey, to examine the developmental trajectories of Chinese adolescent overweigh/obesity, and the potential SES determinants and health consequences of those growth trajectories. Taking into account the great and persisting rural-urban economic, socio-cultural and well-being disparities in China [27], we made explicit comparisons between urban and rural adolescents in the analysis.

\section{Methods}

Data

The data for this study came from four waves of the China Family Panel Studies (CFPS). The CFPS baseline survey was conducted in 2010, and it selected samples from 25 provinces/municipalities/autonomous regions using a PPS sampling method with implicit stratification, representing 95\% of the Chinese population (CFPS User's manual, 3rd edition). The baseline sample was followed up with, biannually in 2012, 2014, and 2016. The 2010 baseline survey interviewed 8990 children aged 0 to 15[1].

For the current analysis, children aged 10 to 12 from the baseline 2010 sample were selected $(\mathrm{N}=1685)$. At the end of the study coverage, they were 16-18 years old in 2016 . This age range corresponds to the biologically critical stage of the adolescent spurt, which occurs between the ages of 10 and 18. Among the original sample, 1388 were reinterviewed in 2012, 1172 in 2014, and 941 in 2016. For the purpose of this study, we retained a final sample of 800 adolescents who had at least three waves of BMI data (i.e. final $\mathrm{N}=800$ ). Combined with the four waves of available data, we have 3200 observations in total.

Measures 
Repeated measures of the observed dependent variables: A binary measure of being overweight or not was used for each of the four waves in 2010, 2012, 2014, and 2016 as our dependent variable. First, adolescent raw BMI scores were computed based on selfreported weights and heights. Second, age- and sex-specific BMI z-scores were calculated according to WHO child growth standards and WHO reference 2007, which were then transformed to age- and sex-specific percentiles for BMI [28]. Finally, according to the CDC cut-points[2], we used the $85^{\text {th }}$ percentile as a cutoff to construct the binary variable of being overweight and obese. Thus, the dependent variable of being overweight or not, included both overweight and obese children in the study.

Predictors at the baseline: We computed all the covariates based on the 2010 baseline data and treated them as time-constant in the modeling specification. First, family SES indicators are our key predictors for class membership of adolescent overweight/obesity. Parental education, family income and parental occupation were used for measuring family SES. Parental education (the higher one between father's and mother's education) was classified into three categories including six years or less (reference), nine years of education, and 12 years or more of education. Family income was divided into low (below $25 \%$ ) (reference), lower medium (25\% to $50 \%$ ), upper medium (50\% to $75 \%$ ), and high (above 75\%) income. Similarly, we categorized parental occupations into four general types including agricultural related jobs (reference), administrative/technical jobs, service/factorial jobs, and other jobs which were missing or unable to be classified.

Subsequent health outcomes: Overall physical health was measured by adolescent selfrated general health in 2016 on a 5-point scale (1-5) with higher scores indicating better self-rated health.

Other covariates that were controlled for include: gender, race, hukou, place of residence (urban vs. rural), family structure (whether living in a two-parents household), average BMI for adult family members, region (East, Middle, West, and Northeast), physical exercise, and breastfeeding type. Furthermore, self-rated health at baseline was 
also included as variables that were controlled for in predicting subsequent health outcomes. Detailed descriptive statistics of these variables can be found in Table 1.

Table 1 Summary Statistics for Key Variables by Place of Residence 


\begin{tabular}{|c|c|c|c|}
\hline & $\begin{array}{l}\text { Urban Area } \\
(\mathrm{N}=303)\end{array}$ & $\begin{array}{l}\text { Rural Area } \\
(\mathrm{N}=497)\end{array}$ & $\begin{array}{l}\text { Full Sample } \\
\qquad(\mathrm{N}=800)\end{array}$ \\
\hline \multirow[t]{2}{*}{ Variables } & Mean (S.E.) & Mean (S.E.) & Mean (S.E.) \\
\hline & / (\%) & / (\%) & / (\%) \\
\hline \multicolumn{4}{|l|}{ Family Income Categories } \\
\hline Low (below 25 \%) (reference) & $16.5 \%$ & $29.18 \%$ & $24.36 \%$ \\
\hline Lower medium (25\% to $50 \%$ ) & $24.09 \%$ & $37.02 \%$ & $32.13 \%$ \\
\hline Upper medium (50 \% to $75 \%$ ) & $30.03 \%$ & $23.34 \%$ & $25.88 \%$ \\
\hline High (above $75 \%$ ) & $29.37 \%$ & $10.46 \%$ & $17.63 \%$ \\
\hline \multicolumn{4}{|l|}{ Parental Education } \\
\hline 6 years or less (reference) & $15.84 \%$ & $46.68 \%$ & $35.00 \%$ \\
\hline 9 years & $42.90 \%$ & $39.24 \%$ & $40.63 \%$ \\
\hline 12 years or more & $41.25 \%$ & $14.08 \%$ & $24.38 \%$ \\
\hline \multicolumn{4}{|l|}{ Parental Occupation } \\
\hline Agricultural related job (reference) & $14.85 \%$ & $53.12 \%$ & $38.63 \%$ \\
\hline Administrative/technical job & $17.49 \%$ & $5.03 \%$ & $9.75 \%$ \\
\hline Service / factorial job & $52.81 \%$ & $31.79 \%$ & $39.75 \%$ \\
\hline Other job & $14.85 \%$ & $10.06 \%$ & $11.88 \%$ \\
\hline Gender $($ male $=1)$ & $53.47 \%$ & $50.30 \%$ & $51.50 \%$ \\
\hline Race $(\operatorname{Han}=1)$ & $91.09 \%$ & $87.73 \%$ & $89.00 \%$ \\
\hline Hukou (Agricultural = 1) & $49.5 \%$ & $95.77 \%$ & $78.25 \%$ \\
\hline \multicolumn{4}{|l|}{ Region } \\
\hline East (reference) & $39.93 \%$ & $19.11 \%$ & $27 \%$ \\
\hline Middle & $26.07 \%$ & $27.36 \%$ & $26.88 \%$ \\
\hline West & $19.14 \%$ & $44.47 \%$ & $34.88 \%$ \\
\hline Northeast & $14.85 \%$ & $9.05 \%$ & $11.25 \%$ \\
\hline Two-Parent Household (Yes = 1) & $90.43 \%$ & $93.16 \%$ & $92.13 \%$ \\
\hline \multicolumn{4}{|l|}{ Physical Exercise } \\
\hline Never (reference) & $21.12 \%$ & $23.34 \%$ & $22.50 \%$ \\
\hline & $7 / 25$ & & \\
\hline
\end{tabular}


Several times a month

Often

Breast Feeding Type

Never (reference)

1 to 6 months

More than 6 months

Average BMI of Adult Family Members

Depression in 2010

Self-Rated Health in 2010

Depression in 2016

Self-Rated Health in 2016
$14.19 \%$

$64.69 \%$

$5.61 \%$

$17.16 \%$

$77.23 \%$

$22.61(2.75)$

$1.41(0.58)$

$4.69(0.63)$

30.26 (5.95)

$3.76(0.99)$
$11.67 \%$

$64.99 \%$

$3.62 \%$

$6.44 \%$

$89.94 \%$

$21.10(3.73)$

21.67 (3.47)

$1.46(0.65)$

$1.44(0.63)$

$4.75(0.54)$

$4.72(0.57)$

30.43 (5.87)

$3.79(0.93)$

\section{Modelling strategy}

Generalized growth mixture modeling (GGMM) [30-31] was used as the major analytical framework in the study. As the first step, latent class growth analysis (LCGA) was used to identify heterogeneity in developmental trajectories of being overweight/obesity among Chinese adolescents. Then a three-step approach [32-33] and BCH methods [34] were adopted to further identify the SES profiles of each subclass and to predict the distal health outcome of class membership. The general model diagram for our analysis is illustrated in Figure 1.

It should be noted that sample attrition is a common issue for longitudinal studies like this. Preliminary examinations showed that attrition was not related with baseline BMI along with many other covariates examined in the study, and this gives us some ground that the assumption of missing at random, which is required by the estimation technique applied in this analysis (i.e. Full Information Maximum Likelihood, FIML), may be a reasonable one to make. 
[Insert Figure 1 here: GGMM Model diagram illustration]

[1] This data set is accessible to any individual who want to use it for a secondary data analysis. CFPS data was collected by a research team from Peking University in China and it has gained required ethic approval for data collection from human subjects. All the related information can be found at their website:

http://www.isss.pku.edu.cn/cfps/index.htm

[2] Although not unanimously agreed, some research suggested that the three major criteria of weight classification (i.e. IOTF, WHO, and CDC) agreed substantially and can be considered comparable in research [29].

\section{Results}

Descriptive Profiles of Adolescent Overweight/Obesity in China

The overall trend of overweight prevalence for Chinese adolescents declined from $23 \%$ to $8 \%$ over time for the studied full sample. However, there exists substantial differences both between gender and place of residence in terms of the prevalence of childhood overweight/obesity. Fig. 2 displays the time trend of the prevalence of overweight by gender and by place of residence (urban/rural) respectively. The prevalence of overweight for boys (from $28 \%$ to $10 \%$ ) is consistently higher than that found for girls (from $18 \%$ to $5 \%$ ) in an almost parallel way over time. The prevalence of overweight for urban children aged $10-12(22 \%)$ is similar to that for rural children (23\%) in 2010. Nonetheless, the prevalence of overweight and obesity for rural adolescents declines steadily faster than that for urban adolescents during the follow-up period. At the age of 16 to 18, the prevalence of overweight for urban adolescents is $10 \%$ while it is $7 \%$ for their rural counterparts.

[Insert Figure 2 here: Prevalence of adolescent overweight by gender and place of residence across time] 
We first estimated latent class growth analysis (LCGA) models that allowed for different numbers of latent classes of overweight trajectories. The corresponding model fit indices are shown in Table 2. Among all the potential models, the three-class solution has the lowest ABIC value and the most reasonable size and shape for each class. The retrieved three classes also make theoretical and substantive sense. Therefore, we will focus on the three-class model for the subsequent analysis[1].

\section{Table 2 Model fit indices for difference number of latent classes}

\begin{tabular}{ccccc}
\hline \# of classes & 1 & 2 & 3 & 4 \\
\hline \# of & 2 & 5 & 8 & 11 \\
parameters & & & & \\
Log likelihood & -1201.880 & -1010.641 & -994.395 & -994.015 \\
BIC & 2417.240 & 2054.979 & 2042.705 & 2062.162 \\
AIC & 2407.761 & 2031.283 & 2004.790 & 2010.029 \\
ABIC & 2410.888 & 2039.101 & 2017.300 & 2027.230 \\
Entropy & -- & 0.919 & 0.636 & 0.652 \\
LMR & -- & $\mathrm{p}=0.0000$ & $\mathrm{p}=0.0012$ & $\mathrm{p}=0.0022$ \\
Class size & $100 \%$ & $91.3 \% /$ & $57.6 \% / 36.4 \% /$ & $50.5 \% / 1.2 \% / 6.3 \% /$ \\
& & $8.7 \%$ & $6.1 \%$ & $42 \%$ \\
\hline
\end{tabular}

As is shown in Fig. 3, we defined Class 1 (57.6\%) as a stable low-risk or normal group, Class $2(36.4 \%)$ as a decreased-risk group, and Class $3(6.1 \%)$ as a sustained high-risk group. For Class 1, the probability of getting overweight is consistently close to zero over time. Class 2 initially has a medium risk of being overweight, which however goes down over time and eventually converges with the first class. In comparison, Class 3 had a consistently high probability (over 90\%) of being overweight over the study period. 
[Insert Fig. 3 here: Heterogeneous developmental trajectories of overweight and obesity for the full sample]

We further estimated the three-class LCGA model separately by gender and by urban/rural division (Fig. 4). Among the sampled girls, only $2.6 \%$ were classified into the stable high-risk group (Class 3 ), while for the boys, it is $9.4 \%$. There are $8.4 \%$ of urban adolescents who were classified into the stable high-risk group (Class 3), while it is only $4.8 \%$ for rural adolescents[2]. This verifies the gender and rural-urban differences in adolescent overweight/obesity that we observed in the above section.

[Insert Figure 4 here: BMI developmental trajectories by place of residence and gender]

SES predictors of class membership

We used the 3-step approach developed by Vermunt (2010) to estimate the association between the predictor variables and the latent class variable. R3STEP option in Mplus was used to specify the auxiliary variables in the model as predictors rather than distal outcomes of the latent class variable. Along with the regular latent class growth model, each observation was assigned to a most likely class variable $\mathrm{N}$ by taking measurement error or classification uncertainty rate into account [3]. In the last step, a multinomial logistic regression model was estimated, in which SES indicators and other controlling covariates predicted the most likely class variable N.

As is mentioned above, the substantial economic and socio-cultural disparities between urban and rural China may lead to fundamental differences in lifestyles, nutrition status, and SES-obesity patterns between urban and rural children. Thus, it was critical to analyze the SES-BMI trajectories separately for both urban and rural adolescents. In fact, in the analysis with the full sample, neither family income nor parental education was found to be 
significantly associated with the latent class membership of BMI trajectories. However, separate analyses for the urban and rural sub-samples revealed more nuances about the potential underlying patterns (Table 3).

Specifically, for the urban sample, since less than one percent of the sampled girls was classified into the sustained high-risk group, we estimated the association between family SES and class memberships only for the urban boys, among whom 14\% was classified into the sustained high-risk group. The results show that higher level of family income in urban area consistently predicts a lower probability of getting into stable high-risk group (ORs = $0.23,0.14$, and 0.23 for the three levels of family income). However, the opposite pattern was found for rural adolescents, in which higher level of family income seemed to increase the probability of stably staying in the high-risk group (ORs $=5.85,4.34$, and 2.66), with only the first income level being statistically significant. In addition, compared to agricultural work, rural adolescents whose parents engage in administrative/technical jobs $(\mathrm{OR}=24.21)$ were also more likely to get into the high-risk class. Further complementary analyses for rural girls and rural boys show similar patterns as reported above.

Table 3 Multinomial Logit Models of Stable High-Risk BMI Class and Normal Class for the Urban and Rural Samples ${ }^{\text {a }}$ 


\begin{tabular}{llllc}
\hline & \multicolumn{2}{c}{ Urban (Male) } & \multicolumn{2}{c}{ Rural } \\
\cline { 2 - 5 } & High vs. Normal & High vs. Normal \\
\hline \multicolumn{1}{c}{ SES Determinants } & O. R. & S. E. & O. R. & S. E. \\
Family Income & & & & \\
[Low income (below $25 \%$ ) as reference] & & & & \\
\hline Lower medium (25\% to $50 \%)$ & $0.23^{+}$ & 0.88 & $5.85^{*}$ & 0.92 \\
Upper medium (50 \% to $75 \%)$ & $0.14^{*}$ & 0.98 & 4.34 & 1.09 \\
\hline High (above $75 \%)$ & $0.23^{+}$ & 0.95 & 2.66 & 1.13
\end{tabular}

Parental Education

[6 years or less of education as reference]

\begin{tabular}{|lllll}
\hline 9 years & 1.45 & 1.24 & 0.54 & 0.75 \\
\hline 12 or more years & 0.99 & 1.45 & 0.54 & 0.92
\end{tabular}

Parental Occupation

[Agricultural related job as reference]

\begin{tabular}{lrrrr}
\hline Administrative/technical job & 3.89 & 1.45 & $24.21^{* *}$ & 1.09 \\
\hline Non-agricultural service / factorial job & 1.59 & 1.28 & 0.56 & 0.85 \\
\hline Other job & 2.02 & 1.16 & 5.77 & 1.60 \\
\hline
\end{tabular}

Note: a. The dependent variable was a three-category nominal variable, but only the comparison between stable high-risk class and normal class was reported in Table 2; b. Results for the control variables in the model was omitted in Table 2; c. ${ }^{+}$p $0.1 ;{ }^{*} p 0.05 ;{ }^{* *} p 0.01 ;{ }^{* * *} p 0.001$.

\section{Health consequences of Adolescent Overweight/Obesity}

We first compared the mean differences among the three classes of overweight/obesity trajectories in adolescents' self-rated health without controlling any covariates. This was 
done by using the automatic $\mathrm{BCH}$ procedure in Mplus. The results show that the sustained high-risk class (class 3) shows significantly lower self-rated health than the decreased risk class. We then further checked these results by including more controls in the model. That is, we predicted the distal outcomes (self-rated health) from the three latent classes while controlling for the effects of other possible confounding variables. This procedure was conducted by using the manual BCH method which estimated a multiple group model with each observation adjusted by a group-specific weight. The class-specific intercepts of selfrated health in 2016 are 3.72 (Class 1), 3.81 (Class 2), and 3.46 (Class 3), after controlling for gender, parental education, family income and baseline self-rated health in 2010. The Wald's Chi-square test shows that the sustained high-risk group (Class 3) had significantly lower self-rated health than the normal group (Class 1) and the decreased-risk group (Class 2).

[1] We also replicated the 3-class solution by an alternative model specification allowing for growth factor variation within each class.

[2] In the complementary analysis, we found that almost all of the $2.6 \%$ of the sampled girls who were classified into Class 3 are from rural areas, whereas a much higher proportion of urban boys were classified into Class 3 than rural boys (14.1\% vs. $6.7 \%$ ).

[3] $\mathrm{N}$ is treated as an imperfect indicator of the latent class variable $\mathrm{C}$ with measurement error defined by the logits for the classification probabilities for most likely class membership [33].

\section{Discussion}

This study is among the first that specifically focuses on the developmental trajectories of adolescent overweight/obesity in China. Based on a nationally representative longitudinal survey, we identified three heterogeneous classes of developmental trajectories for adolescent BMI, and examine their associated SES precursors and subsequent health outcomes. Three major findings from our study are worthy of further attention and discussion. 
First, the three classes of BMI developmental trajectories identified in our study are not identical to those typically found in Western societies. In previous studies on Western children, the proportion of consistently normal is higher and a late onset class is usually identified [17-18]. Given the data-driven nature of latent class analysis, further research should examine these disparities with different and larger representative data of Chinese adolescents.

Second, regarding the association between family SES indicators and adolescent BMI trajectory types, the most critical finding in our study is that the effect of family income on adolescent overweight/obesity is in the opposite direction for urban and rural China. That is, higher family income in urban areas seems to protect urban boys from the risk of sustained overweight/obesity, while it increases the same risk for rural adolescents. Furthermore, we found that for rural adolescents, the effect of parental occupation is consistent with that of family income. The social stratification research on contemporary China suggests that rural residents who live purely on agricultural income has become the lowest class in current rural society, while those who occupy village administrative positions (the so-called "rural cadres") has become the highest class [35]. Taken together, our findings suggest that urban China is ahead of rural China in terms of nutrition transition. We speculate that urban China on average may have entered into the "behavioral change" stage while the rural areas on average are still experiencing the "degenerative diseases" stage[1] according to the nutrition transition theory [1]. But this speculation should be interpreted with caution and further research.

Third, we found that adolescents who are chronically overweight/obese reported significantly lower self-rated health. In other words, negative physical health consequence may have started emerging for these young people. However, given the limitation of data, we are unable to examine more specific health consequences of childhood overweight/obesity[2]. Future research should investigate the association between BMI trajectories with specific types of health problems, which may be highly likely to occur during adolescence and early adulthood, such as asthma [22]. 
Although our research findings are quite enlightening, there are limitations that should be acknowledged. First, our sample was selected from a nationally representative data set but the sample size of adolescents was relatively small, which may have hampered our capability of detecting more complete and accurate patterns of adolescent overweight/obesity in China. Second, our BMI data were based on self-reported weights and heights[3], which might lead to some systematic bias in the estimation of overweight prevalence. The accuracy of self-reported height and weight is greatly debated in the literature [36-38]. Third, research has shown that rural adolescents in China are experiencing double burdens of both under-nutrition and over-nutrition [39]. In this study, we focused only on the issue of overweight/obesity and its correlates, and thus do not distinguish being normal from being underweight. However, child underweight in China may be worthy of special attention.

[1] The "degenerative disease" pattern is characterized with a diet high in total fact, sugar, and cholesterol etc., accompanied by a sedentary life style and thus resulting in increased prevalence of obesity and related degenerative disease. The "behavioral change" pattern is characterized with a change toward a healthier life style such as less fat and processing food and more exercise to delay generative disease and promote health (Popkin, 1993).

[2] In our sample, only 27 children reported some kind of chronic disease in 2016. Seven of them were upper respiratory tract infection and eight were gastroenteritis.

[3] From wave 1 to wave 3, parents reported their children's weights and heights, and in wave 4 the adolescents reported their own anthropometric data.

\section{Conclusions}

This study provides some evidence on the potential overweight/obesity developmental trajectories as well as SES determinants and subsequent health consequence of those growth trajectories among Chinese adolescents, which has been largely absent in the current literature. Despite the limitations discussed above, this study still sheds light on the complicated patterns of SES-BMI growth trajectories among Chinese adolescents during the great social transformation era in China. The overweight prevalence of urban 
adolescents is much higher than rural children, which implies that more economically developed areas need more intense interventions on this public health threat. Moreover, in developing effective interventions, different risk factors should be considered for urban and rural areas in that the SES correlates of overweight/obesity seem to be quite opposite for urban and rural adolescents. While poverty in urban areas is a risk factor for adolescent overweight, being rich in rural areas may be equally dangerous for childhood overweight. Therefore, we argue that the economic division between urban and rural China is a fundamental force in shaping different stages of nutrition transition and leads to different associations between SES and children's BMI trajectories. Contemporary China is not experiencing one single nutrition transitional stage but rather a mixture of nutrition transition. In this regard, it is crucial to identify context-specific public health threats and develop strategic counteracts accordingly.

\section{Declarations}

\section{Abbreviations}

CFPS: China Family Panel Studies; CDC: Centers for Disease Control and Prevention; GGMM: Generalized growth mixture modeling; IOTF: International Obesity Task Force; SES: Socioeconomic Status; WHO: the World Health Organization;

\section{Acknowledgements}

Not applicable.

\section{Authors' contributions}

YL was responsible for the overall study design, part of literature search and summary, data analysis and manuscript development. YQQ was responsible for part of literature search and writing, some complementary data analysis and some final revisions of the manuscript. All authors read and approved the final manuscript. 


\section{Funding}

This work was supported by the Liberal Arts and Social Sciences Fund of Ministry of

Education in China (MOE) [Grant Number 19YJC840021], and by the Fundamental

Research Funds for the Central Universities in China.

\section{Availability of data and materials}

CFPS data is publicly available on its website: http://www.isss.pku.edu.cn/cfps/. The subdata set used in the current study is available from the corresponding author on request.

\section{Ethics approval and consent to participate}

Not required owing to the design of the research (a secondary analysis of de-identified public data)

Consent for publication

Not applicable.

Competing interests

The authors declare that they have no conflict of interest

\section{References}

1. Popkin BM. Nutritional patterns and transitions. Population and Development Review 1993; 19: 138-157.

2. Popkin BM, Doak CM. The obesity epidemic is a worldwide phenomenon. Nutrition Reviews 1998; 56: 106-114.

3. Popkin BM. Will China's nutrition transition overwhelm its health care system and slow economic growth? Health Affairs 2008; 27: 1064-1076.

4. Zhai F, Wang H, Du S, et al. Prospective study on nutrition transition in China. Nutrition Reviews 2009; 67: S56-S61.

5. Ji CY, Cooperative Study on Childhood Obesity: Working Group on Obesity in China (WGOC). The prevalence of childhood overweight/obesity and the epidemic changes in 1985-2000 for Chinese school-age children and adolescents. Obesity Reviews 2008; 9: S78-S81. 
6. Jia P, Xue H, Zhang J, Wang Y. Time trend and demographic and geographic disparities in childhood obesity prevalence in China-evidence from twenty years of longitudinal data. International J of Environmental Research and Public Health 2017; 14: 369.

7. Must A, Strauss RS. Risks and consequences of childhood and adolescent obesity. International J of Obesity 1999; 23(S2).

8. Franks PW, Hanson RL, Knowler WC, et al. Childhood obesity, other cardiovascular risk factors, and premature death. New England Journal of Medicine 2010;362: 485493.

9. Reilly JJ, Kelly J. Long-term impact of overweight and obesity in childhood and adolescence on morbidity and premature mortality in adulthood: systematic review. International J of Obesity 2011, 35: 891.

10. Sobal J, Stunkard AJ. Socioeconomic status and obesity: a review of the literature. Psychological Bulletin 1989, 105: 260.

11. Shrewsbury V, Wardle J. Socioeconomic status and adiposity in childhood: a systematic review of cross-sectional studies 1990-2005. Obesity 2008; 16: 275-284.

12. Ogden CL, Lamb MM, Carroll MD, Flegal KM. Obesity and Socioeconomic Status in Children and Adolescents: United States, 2005-2008. NCHS Data Brief. Number 51. National Center for Health Statistics 2010.

13. Monteiro CA, Moura EC, Conde WL, Popkin BM. Socioeconomic status and obesity in adult populations of developing countries: a review. Bulletin of the World Health Organization 2004; 82: 940-946.

14. Dinsa GD, Goryakin Y, Fumagalli E, Suhrcke M. Obesity and socioeconomic status in developing countries: a systematic review. Obesity Reviews 2012; 13: 1067-1079.

15. Cui Z, Huxley R, Wu Y, Dibley MJ. Temporal trends in overweight and obesity of children and adolescents from nine Provinces in China from 1991-2006. International J of Pediatric Obesity 2010; 5: 365-374.

16. He W, James SA, Merli MG, Zheng H. An increasing socioeconomic gap in childhood overweight and obesity in China. American J of Public Health 2014; 104: e14-e22.

17. Mustillo S, Worthman C, Erkanli A, et al. Obesity and psychiatric disorder: developmental trajectories. Pediatrics 2003; 111: 851-859.

18. Li C, Goran MI, Kaur H, et al. Developmental trajectories of overweight during childhood: role of early life factors. Obesity 2007; 15: 760-771.

19. Balistreri KS, Van Hook J. Trajectories of overweight among US school children: a focus on social and economic characteristics. Maternal and Child Health J 2011; 15: 610-619.

20. Lane SP, Bluestone C, Burke CT. Trajectories of BMI from early childhood through early adolescence: SES and psychosocial predictors. British J of Health Psychology 2013; 18: 66-82. 
21. Howe LD, Tilling K, Galobardes B, et al. Socioeconomic disparities in trajectories of adiposity across childhood. International J of Pediatric Obesity 2011; 6(sup3): e144153.

22. Ziyab AH, Karmaus W, Kurukulaaratchy RJ, et al. Developmental trajectories of Body Mass Index from infancy to 18 years of age: prenatal determinants and health consequences. J Epidemiol Community Health 2014; 68: 934-941.

23. Ventura AK, Loken E, Birch LL. Developmental trajectories of girls' BMI across childhood and adolescence. Obesity 2009; 17: 2067-2074.

24. Sanders RH, Han A, Baker JS, Cobley S. Childhood obesity and its physical and psychological co-morbidities: a systematic review of Australian children and adolescents. European J of Pediatrics 2015; 174: 715-746.

25. Pulgaron ER. Childhood obesity: a review of increased risk for physical and psychological comorbidities. Clinical Therapeutics 2013; 35: A18-A32.

26. Xie Y, Zhou X. Income inequality in today's China. Proceedings of the National Academy of Sciences 2014; 111: 6928-6933.

27. Treiman DJ. The "difference between heaven and earth": Urban-rural disparities in well-being in China. Research in Social Stratification and Mobility 2012; 30: 33-47.

28. Vidmar SI, Cole TJ, Pan H. Standardizing anthropometric measures in children and adolescents with functions for egen: Update. The Stata Journal 2013; 13: 366-378.

29. Li K, Haynie D, Palla H, et al. Assessment of adolescent weight status: Similarities and differences between CDC, IOTF, and WHO references. Preventive Medicine 2016; 87: 151-154.

30. Muthén BO. Beyond SEM: General latent variable modeling. Behaviormetrika 2002; 29: 81-117.

31. Muthén BO. Latent variable analysis: growth mixture modeling and related techniques for longitudinal data. In Kaplan D, eds. The Sage Handbook of Quantitative Methodology for the Social Sciences. Sage, 2004: 345-368.

32. Vermunt JK. Latent class modeling with covariates: Two improved three-step approaches. Political Analysis 2010; 18: 450-469.

33. Asparouhov T, Muthén BO. Auxiliary variables in mixture modeling: Three-step approaches using Mplus. Structural Equation Modeling: A Multidisciplinary Journal 2014a; 21: 329-341.

34. Asparouhov T, Muthén BO. Auxiliary variables in mixture modeling: Using the $\mathrm{BCH}$ method in Mplus to estimate a distal outcome model and an arbitrary secondary model. Mplus Web Notes 2014b; 21(2): 1-22.

35. Bian Y. Chinese social stratification and social mobility. Annual review of sociology 2002; 28: 91-116. 
36. Dubois L, Girad M. Accuracy of maternal reports of pre-schoolers' weights and heights as estimates of BMI values. International J of Epidemiology 2007; 36: 132-138.

37. Craig BM, Adams AK. Accuracy of body mass index categories based on self-reported height and weight among women in the United States. Maternal and Child Health J 2009; 13: 489-496.

38. Gosse MA. How accurate is self-reported BMI? Nutrition bulletin 2014; 39: 105-114.

39. Zhang N, Bécares L, Chandola T. Patterns and determinants of double-burden of malnutrition among rural children: evidence from China. PloS One 2016; 11.

\section{Figures}




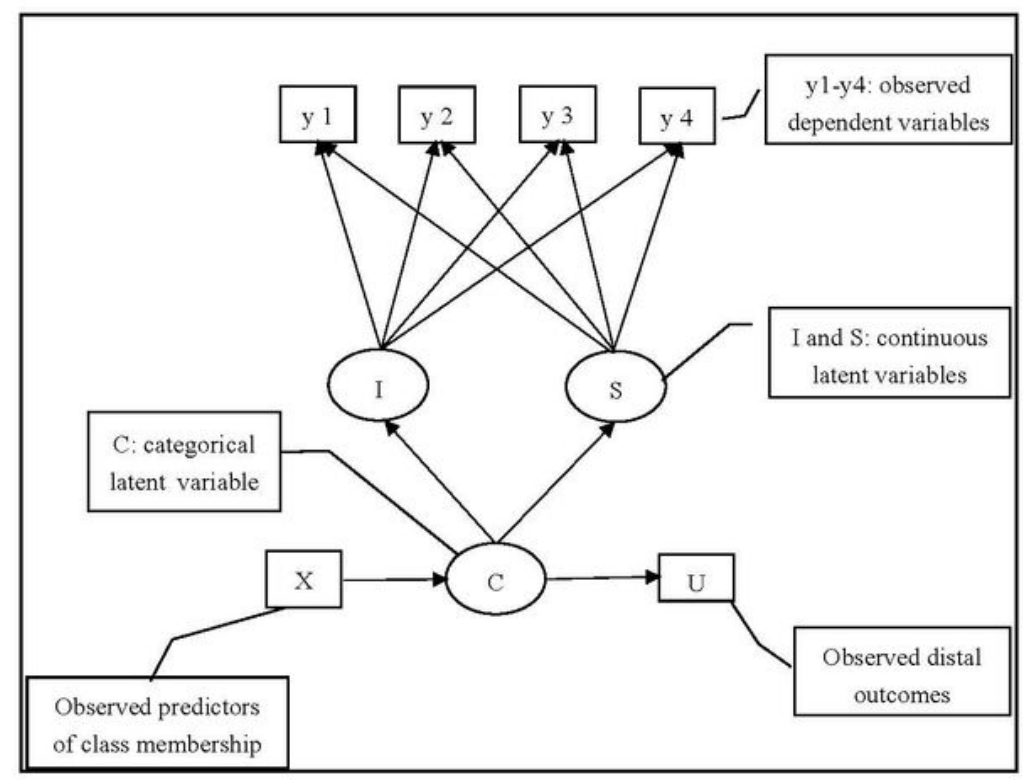

Figure 1. GGMM Model diagram illustration

Figure 1

GGMM Model diagram illustration 

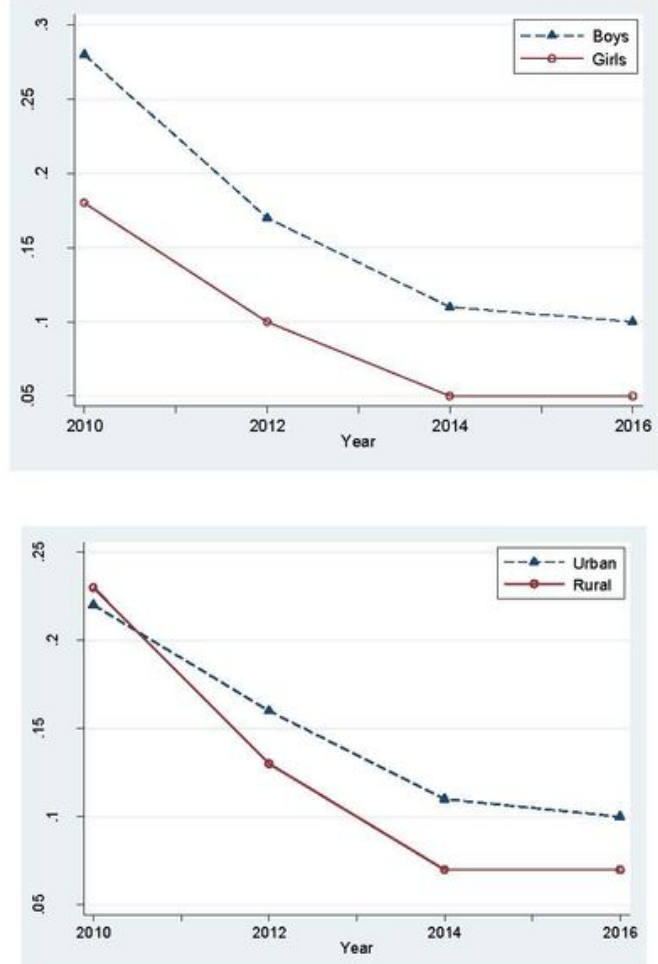

Figure 2. Prevalence of adolescent overweight by gender and place of residence across time

Figure 2

prevalence of adolescent overweight by gender and place of residence across time 


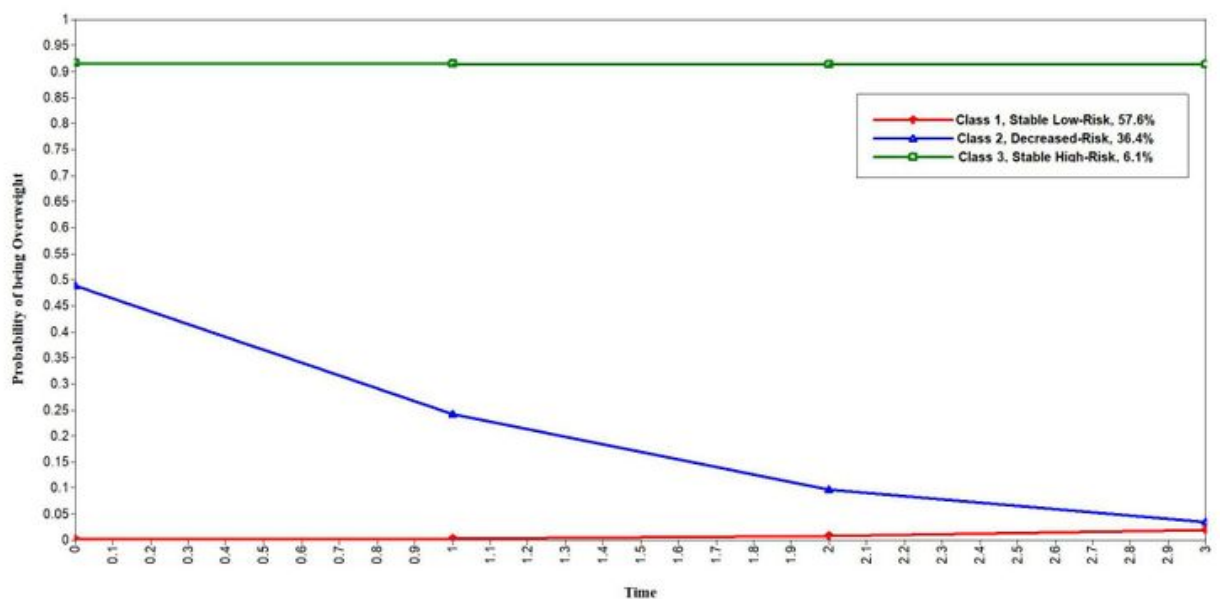

Figure 3. Heterogeneous developmental trajectories of overweight and obesity for the full sample

\section{Figure 3}

Heterogeneous developmental trajectories of overweight and obesity for the full sample 


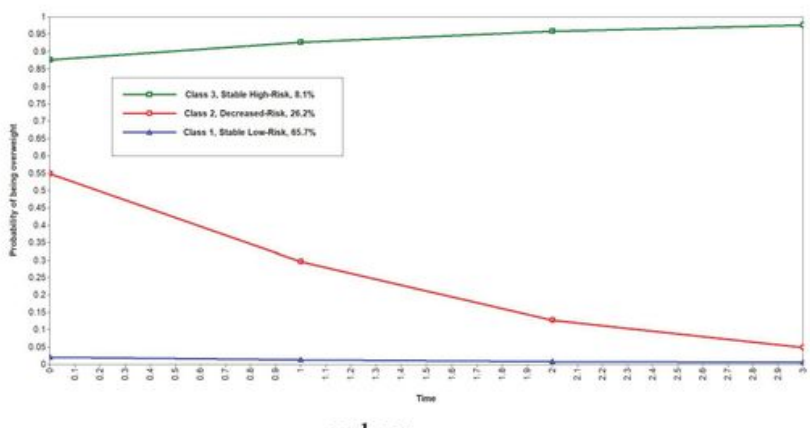

urban

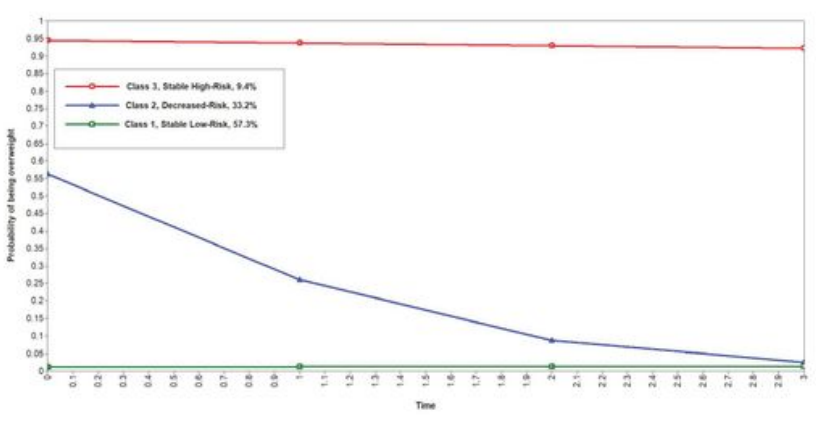

Boys

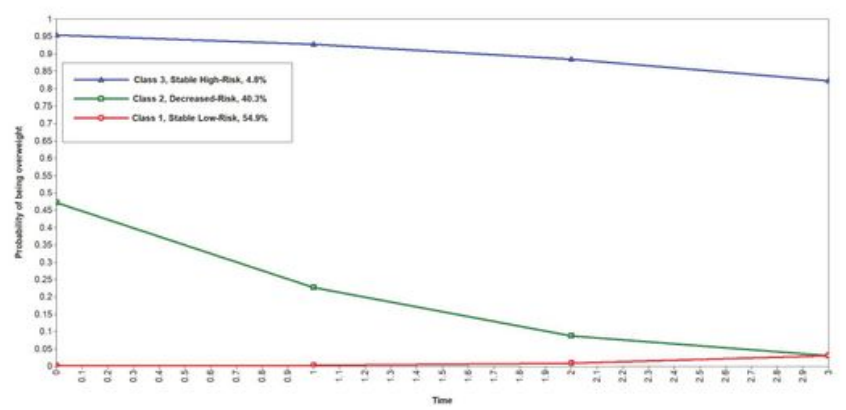

rural

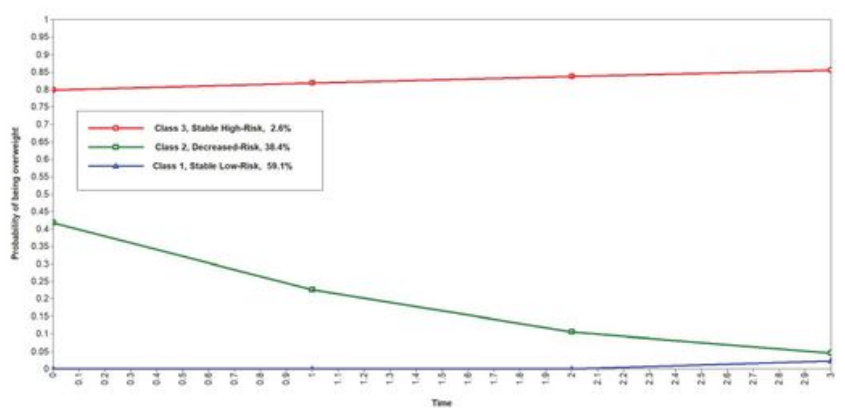

Girls

Figure 4. Heterogeneous developmental trajectories of overweight and obesity by place of residence and by gender

\section{Figure 4}

BMI developmental trajectories by place of residence and gender 